\title{
Effect of Doping Concentration on STRUCTURAL Stability and Formation Energy of the Fluorine Doped Hexagonal Molybdenum Dioxide $\left(\mathrm{MoO}_{2}\right)$. A First Principle Study
}

\author{
Yakubu A Tanko ${ }^{1}$, Alhassan Shuaibu ${ }^{1}$, Aminu Abdulrahman ${ }^{1}$, Oyedare P Olusola ${ }^{2}$, \\ Mustapha Isa ${ }^{1}$ and Aminu S Yamusa ${ }^{3}$ \\ ${ }^{1}$ Department of physics Faculty of science Kaduna state university \\ ${ }^{2}$ Department of Science Laboratory Technology, Federal Polytechnic Ede \\ ${ }^{3}$ Department of Physics, Federal college of Education Zaria \\ Corresponding E-mail: aminuabdulrahman72@gmail.com
}

Received 21-05-2021

Accepted for publication 29-06-2021

Published 13-08-2021

\begin{abstract}
The structural properties of undoped and Fluorine doped Hexagonal Molybdenum dioxide $\left(\mathrm{MoO}_{2}\right)$ with different doping concentrations have been calculated using Density Functional Theory (DFT) within Generalized Gradient Approximation (GGA) as implemented in Quantum Espresso (QE). The calculated results were for the formation energy of $4.17 \%, 8.33 \%, 12.5 \%$, of $\mathrm{F}$ doped $\mathrm{MoO}_{2}$ are $232.5 \mathrm{eV}, 463.0 \mathrm{eV}$, and $698.5 \mathrm{eV}$ respectively, which show the variation of energy based on the increase in the doping concentration that led to having the breakage of bond in the structure of the compound. The undoped and $4.17 \%$ of $\mathrm{F}$ doped $\mathrm{MoO}_{2}$ have three free atoms, which maintain the stability of the structure, but when the doping concentration was increased, the bond breaks simultaneously which led to having four and five free atoms for $8.33 \%$, and $12.5 \%$ of $\mathrm{F}$ doped $\mathrm{MoO}_{2}$ respectively. This makes $4.17 \%$ of $\mathrm{F}$ doped $\mathrm{MoO}_{2}$ with 17.09Ry more stable. Similarly, the bond length of undoped $\mathrm{MoO}_{2}$ was $2.2505 \mathrm{pm}$, but when doped with $4.17 \%$ of $\mathrm{F}$ it changes to $2.3030 \mathrm{pm}$ which indicates a greater stability of the structure concentrations of the dopant above $4.17 \%$ reduced the bond length, which made the structure less stable.
\end{abstract}

Keywords: Fluorine; Doped; Density functional theory; generalized gradient approximation; Quantum ESPRESSO

\section{INTRODUCTION}

$\mathrm{V}$ arious 2D materials such as graphene, transition metal dichalcogenides, antimony, black phosphorus, have been synthesized [1-6] to illustrate the incredible potential for new type of optoelectronic devices. owing to their unique properties and rich in feasibility for the fabrication of $2 \mathrm{D}$

VOLUME 01, ISSUE 01, 2021 materials technologies [7]. Certainly, some of specific 2D materials have shortcomings such as zero band gap, low absorption efficiency, and instability in open atmosphere which are some of the challenges in fabrication of ideal nanoscale devices. In order to overcome these challenges, the transition metal oxides TMO have been found to be effective 2D materials in terms of possessing high conductivity, piezoelectricity, colossal magnetoresistance, better stability in 
open environment and superconductivity, etc. [8-10]. Molybdenum dioxide is a typical TMO material having three crystalline polymorphic forms; hexagonal phase $\left(\mathrm{P}_{3} / m m c\right)$ [11], tetragonal phase $\left(\mathrm{P}_{2} / \mathrm{mnm}\right)$ [12] and monoclinic $\left(\mathrm{P} 2_{1} / \mathrm{c}\right)$ [13], and also possesses a partially rutile configuration [14] containing $\mathrm{MoO}_{6}$, octahedrally linked Mo through oxygen atoms in the edges of unit cell involves four $\mathrm{MoO}_{2}$ units which is opposite to two-unit cells [15-16], It is well known that the properties of molybdenum oxides are strongly dependent on their crystalline structures; in particular, the rutile structure of $\mathrm{MoO}_{2}$ is interesting due to possession of superb metallic-like electrical conductivity, low electrical resistivity, high melting point [17-18], facile ion transport [19], and excellent chemical stability [20]. It has been interrelated to various interatomic bonding and comparatively over the top density of states at the Fermi level. The presence of free electrons generates $\mathrm{Mo}^{4+}$ in $\mathrm{MoO}_{2}$ in contrast to generation of $\mathrm{Mo}^{6+}$ from $\mathrm{MoO}_{3}$; hence, all the valence electrons in molybdenum metal are covalently bonded to nearest oxygen atoms [21]. A small variation in Mo valence may cause significant fluctuations in physical properties of the molybdenum oxides. For instance, it is possible to obtain compounds of other oxides with diverse physical properties [22-23].

The main difference of transition metals (TM) from other metals is that their valence electrons may be present in more than one shell. Hence, most TM have more than one oxidation states. The oxides of TM show a rich variety of electronic properties, ranging from insulating to metallic and even superconducting behavior. The same applies to their magnetic properties, where everything is found from Pauli Paramagnetic to local moment behavior including the occurrence of ferromagnetism and anti-ferromagnetism. Additionally, these materials can often be tuned from one electronic or magnetic phase to another by varying the temperature, pressure, or by doping. Therefore, the transition metal oxides TMO have been for a long time the subject of intense experimental and theoretical study. Especially d-band TMO are of interest because of their catalytic properties. Transition metal oxides are used in a wide variety of technologically important catalytic processes. For example, they are used in selective oxidation, selective reduction and dehydrogenation [24]. Understanding surface structure and reactions is important for understanding these catalytic processes.

Theoretically and experimentally, Hexagonal $\mathrm{MoO}_{2}$ has received less attention than other structural phases of $\mathrm{MoO}_{2}$, with only a handful of studies in the literature [25-27]..This is because the Hexagonal phase has major problem of metallic character and weak paramagnetic properties at room temperature [28], Recently two studies by Eyert and coworkers used LDA within the augmented spherical wave (ASW) method to investigate the instability in the Hexagonal $\mathrm{MoO}_{2}$ and also to study the Fermi surface of $\mathrm{MoO}_{2}$ in comparison with angle-resolved photoemission spectroscopy.

Over the past few decades, other crystal phases of $\mathrm{MoO}_{2}$ have been utilized in electrocatalysts application, these studies have indicated that the activity of crystal size in Hexagonal $\mathrm{MoO}_{2}$ correlates with the number of the unsaturated $\mathrm{Mo}$ and $\mathrm{O}$ sites along the edges, where as their basal planes are catalytically passive.

Therefore, exposing the maximum number of edge sites and improving the intrinsic activity of the edge sites of the hexagonal $\mathrm{MoO}_{2}$ by chemical doping have become main strategies to enhance the activity.

\section{COMPUTATIONAL DETAILS}

DFT calculations were performed using the project augmented wave basis set as implemented in Quantum ESPRESSO. [29] A plane wave cut-off energy was used as $160 \mathrm{eV}$ was used, and the exchange correlation function was treated using the PBE for the GGA [30]. The valence electrons of Molybdenum (Mo) and Oxygen were $4 d^{5} 5 s^{1}$ and $2 s^{2} 2 p^{6}$ respectively [31] with hexagonal structure, space group $p 6_{3} / \mathrm{mmc}\{194\}$, and point group $6 / \mathrm{mmm}$. A simple conventional unit cell with lattice parameters of: $\mathrm{a}=\mathrm{b}=2.859 \AA, \mathrm{c}=10.460 \AA, \propto=90^{\circ}=\beta$ and $\gamma=120.001^{\circ}$ contains only two Molybdenum (Mo) and four Oxygen atoms $(\mathrm{O})$, a supercell of $2 \times 2 \times 1$ is used in order to increase the number of atoms relative to the standard conventional unit cell for both the pure and doped Molybdenum dioxide. The supercell consists of twenty-four (24) atoms with eight (8) Molybdenum and sixteen (16) Oxygen satom, the new lattice parameters for the supercells of Molybdenum dioxide are $a=4.951119 \hat{A}=b$, and $c=$

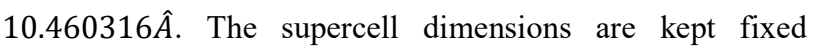
throughout the calculations, while the atomic positions are fully relaxed for all calculations using Broyden FletcherGoldfarb-Shannon (BFGS) algorithm, until the forces acting on the atoms are below 0:001 eV/Å.

\section{RESULT AND DISCUSSION}

\section{A. Structural Properties}

Define Table I is the Substitutional configuration of $4.17 \%$ of fluorine doped Hexagonal Molybdenum dioxide, there are sixteen (16) different symmetrical approach in which one 
oxygen can be replaced by one fluorine in different doping concentrations [32]. in present study $D_{16} 0.0417$ was considered. The structure is more stable than the pure Molybdenum dioxide which both have three free atoms, and there is no transition between the structures and the crystals parameters remain the same as that of the pure (undoped) Molybdenum dioxide [33].

TABLE I CONFIGURATION FOR SUBSTITUTIONAL DOPING OF $4.17 \%$ OF FLUORINE ON OXYGEN IN MOLYBDENUM DIOXIDE

\begin{tabular}{|c|c|c|c|c|c|c|c|c|c|c|c|c|c|c|c|c|}
\hline & & & \multicolumn{14}{|c|}{ MOLYBDENUM DIOXIDE } \\
\hline Jndoped & $\mathbf{o}$ & $\mathrm{o}$ & $\mathbf{o}$ & $\mathbf{o}$ & o & $\mathbf{o}$ & & $\mathbf{O}$ & $\mathbf{o}$ & & $\mathbf{O}$ & $\mathbf{O}$ & $\mathbf{o}$ & $\mathbf{O}$ & $\mathbf{O}$ & $\mathbf{o}$ \\
\hline$\overline{D_{1} \mathbf{0 . 0 4 1 7}}$ & F & $\begin{array}{ll}0 \\
\end{array}$ & \begin{tabular}{ll|l}
$\mathbf{o}$ & \\
\end{tabular} & $\begin{array}{l}\mathbf{O} \\
\end{array}$ & $\begin{array}{l}\mathbf{0} \\
\end{array}$ & $\begin{array}{l}\mathbf{O} \\
\end{array}$ & $\begin{array}{ll}0 \\
\end{array}$ & $\mathbf{0}$ & $\begin{array}{l}\mathbf{0} \\
\end{array}$ & $\begin{array}{l}\mathbf{O} \\
\end{array}$ & 0 & 0 & $\begin{array}{ll}\mathbf{O} \\
\end{array}$ & 0 & $\mathbf{0}$ & \begin{tabular}{|l|l|l|}
0 & \\
\end{tabular} \\
\hline$D_{2} 0.0417$ & $\mathbf{O}$ & $\mathbf{F}$ & $\mathbf{o}$ & $\mathbf{o}$ & $\mathbf{o}$ & $\mathbf{o}$ & $\mathrm{o}$ & $\mathbf{O}$ & $\mathbf{o}$ & 0 & 0 & 0 & $\mathrm{o}$ & o & $\mathbf{0}$ & $\mathbf{o}$ \\
\hline$D_{3} 0.0$ & 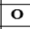 & $\mathrm{o}$ & $\mathbf{F}$ & o & $\mathbf{o}$ & 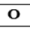 & o & $\mathbf{o}$ & $\mathbf{o}$ & o & o & $\mathbf{O}$ & $\mathbf{o}$ & o & o & o \\
\hline$D_{4} 0.0417$ & $\mathbf{o}$ & $\mathrm{o}$ & $\mathbf{o}$ & $\mathbf{F}$ & $\mathbf{o}$ & $\mathbf{o}$ & $\mathrm{o}$ & $\mathbf{o}$ & $\mathbf{o}$ & $\mathbf{o}$ & o & $\mathbf{0}$ & $\mathrm{o}$ & o & o & $\mathbf{o}$ \\
\hline$D_{5} \mathbf{0 . 0 4 1 7}$ & $\mathbf{0}$ & 0 & $\mathbf{0}$ & $\mathbf{0}$ & $\mathbf{F}$ & $\mathbf{o}$ & $\mathbf{o}$ & $\mathbf{O}$ & $\mathbf{0}$ & $\mathbf{o}$ & $\mathbf{O}$ & $\mathbf{O}$ & $\mathbf{O}$ & $\mathbf{O}$ & $\mathbf{0}$ & o \\
\hline$D_{6} 0.0417$ & $\mathbf{0}$ & 0 & $\mathbf{0}$ & o & o & $\mathbf{F}$ & 0 & $\mathbf{O}$ & $\mathbf{O}$ & o & 0 & 0 & 0 & 0 & 0 & 0 \\
\hline$D_{7} 0.0417$ & 0 & 0 & $\mathbf{0}$ & 0 & o & 0 & $\mathbf{F}$ & o & o & o & 0 & 0 & 0 & 0 & o & 0 \\
\hline$D_{8} 0.0417$ & o & 0 & o & o & o & o & 0 & $\mathbf{F}$ & o & o & 0 & 0 & o & 0 & o & 0 \\
\hline$D_{9} \mathbf{0 . 0 4 1 7}$ & o & o & $\mathbf{o}$ & o & o & $\mathbf{o}$ & o & $\begin{array}{l}\mathbf{O} \\
\end{array}$ & $\mathbf{F}$ & $\mathbf{o}$ & $\mathbf{O}$ & $\mathbf{O}$ & $\mathbf{0}$ & $\mathbf{O}$ & \begin{tabular}{|l|l|}
$\mathbf{O}$ \\
\end{tabular} & o \\
\hline$D_{10} 0.0417$ & $\mathbf{0}$ & 0 & 0 & 0 & o & 0 & 0 & 0 & 0 & $\mathbf{F}$ & 0 & 0 & 0 & 0 & $\mathbf{0}$ & 0 \\
\hline$D_{11} 0.0417$ & $\mathbf{0}$ & 0 & $\mathbf{o}$ & o & 0 & 0 & 0 & 0 & 0 & 0 & $\mathbf{F}$ & 0 & 0 & 0 & 0 & 0 \\
\hline$D_{12} 0.0417$ & o & $\mathrm{o}$ & 0 & $\mathrm{o}$ & 0 & o & $\mathrm{O}$ & o & o & 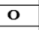 & 0 & $\mathbf{F}$ & o & $\mathrm{O}$ & & o \\
\hline$D_{14} 0.0417$ & o & 0 & $\mathbf{0}$ & o & o & o & 0 & o & $\mathbf{o}$ & o & 0 & 0 & o & F & o & o \\
\hline$D_{15} 0.0417$ & 0 & 0 & $\mathbf{0}$ & 0 & o & 0 & 0 & $\mathbf{0}$ & $\mathbf{0}$ & 0 & 0 & 0 & 0 & 0 & F & o \\
\hline$D_{16} 0.0417$ & o & o & $\mathbf{0}$ & o & o & o & o & o & o & o & 0 & 0 & o & 0 & $\mathbf{0}$ & F \\
\hline
\end{tabular}

Similarly, table II is the Substitutional configuration of $8.33 \%$ of fluorine doped Hexagonal Molybdenum dioxide, there are eight (8) different symmetrical approach in which two oxygen can be replaced by two fluorine in different doping concentrations. in present study $D_{1} 0.0833$ was considered [32]. The structure is not stable and there are four free atoms in the structure, but the crystals parameters remain the same as that of the pure (undoped) Molybdenum dioxide [33].

TABLE II CONFIGURATION FOR SUBSTITUTIONAL DOPING OF 8.33\% OF FLUORINE ON OXYGEN IN

\begin{tabular}{|c|c|c|c|c|c|c|c|c|c|c|c|c|c|c|c|c|}
\hline \multicolumn{17}{|c|}{ MOLYBDENUM DIOXIDE } \\
\hline Undoped & $\mathbf{O}$ & $\mathbf{O}$ & $\mathbf{o}$ & $\mathbf{o}$ & $\mathbf{o}$ & $\mathbf{o}$ & $\mathbf{o}$ & $\mathbf{o}$ & $\mathbf{o}$ & $\mathbf{o}$ & $\mathbf{o}$ & $\mathbf{O}$ & $\mathbf{o}$ & $\mathbf{O}$ & o & $\mathbf{o}$ \\
\hline$D_{1} 0.0833$ & $\mathbf{F}$ & $\mathbf{o}$ & $\mathbf{o}$ & o & o & $\mathbf{o}$ & o & o & $\mathbf{o}$ & $\mathbf{o}$ & o & o & $\mathbf{o}$ & o & o & $\mathbf{F}$ \\
\hline$D_{2} \mathbf{0 . 0 8 3 3}$ & o & $\mathbf{F}$ & $\mathbf{o}$ & $\mathbf{O}$ & $\mathbf{O}$ & $\mathbf{o}$ & $\mathbf{o}$ & $\mathbf{O}$ & $\mathbf{o}$ & $\mathbf{0}$ & $\mathbf{o}$ & $\mathbf{O}$ & $\mathbf{O}$ & $\mathbf{O}$ & $\mathbf{F}$ & $\mathbf{o}$ \\
\hline$D_{3} 0.0833$ & $\mathbf{o}$ & $\mathbf{o}$ & $\mathbf{F}$ & $\mathbf{O}$ & $\mathbf{O}$ & $\mathbf{o}$ & $\mathbf{o}$ & $\mathbf{O}$ & $\mathbf{o}$ & $\mathbf{o}$ & $\mathbf{o}$ & $\mathbf{O}$ & $\mathbf{O}$ & $\mathbf{F}$ & o & $\mathbf{o}$ \\
\hline$D_{4} \mathbf{0 . 0 8 3 3}$ & 0 & $\mathbf{o}$ & $\mathbf{0}$ & $\mathbf{F}$ & $\mathbf{O}$ & $\mathbf{o}$ & o & $\mathbf{O}$ & $\mathbf{o}$ & $\mathbf{o}$ & $\mathbf{O}$ & 0 & $\mathbf{F}$ & 0 & 0 & 0 \\
\hline$D_{5} \mathbf{0 . 0 8 3 3}$ & 0 & o & o & o & $\mathbf{F}$ & o & o & o & o & o & o & $\mathbf{F}$ & o & o & o & $\mathbf{o}$ \\
\hline$D_{6} 0.0833$ & o & o & o & o & O & $\mathbf{F}$ & o & O & O & O & F & O & 0 & o & o & o \\
\hline$D_{7} \mathbf{0 . 0 8 3 3}$ & o & 0 & 0 & 0 & o & O & $\mathbf{F}$ & O & o & $\mathbf{F}$ & 0 & O & 0 & 0 & 0 & o \\
\hline$D_{8} 0.0833$ & 0 & 0 & 0 & 0 & 0 & O & o & $\mathbf{F}$ & $\mathbf{F}$ & O & 0 & 0 & 0 & 0 & 0 & 0 \\
\hline
\end{tabular}

Also, table III is the Substitutional configuration of $12.5 \%$ of fluorine doped Hexagonal Molybdenum dioxide, there are fourteen (14) different symmetrical approach in which three oxygen can be replaced by three fluorine in different doping concentrations. in present study $D_{8} 0.125$ configuration was considered [32]. The structure is not stable and there are five free atoms in the structure, but the crystals parameters remain the same as that of the pure (undoped) Molybdenum dioxide [33].
TABLE III CONFIGURATION FOR SUBSTITUTIONAL DOPING OF $12.5 \%$ OF FLUORINE ON OXYGEN IN MOLYBDENUM DIOXIDE

\begin{tabular}{|c|c|c|c|c|c|c|c|c|c|c|c|c|c|c|c|c|}
\hline Undoped & 0 & 0 & 0 & 0 & 0 & 0 & 0 & 0 & 0 & 0 & 0 & 0 & 0 & 0 & 0 & 0 \\
\hline$D_{1} 0.125$ & F & F & 0 & 0 & 0 & 0 & 0 & 0 & 0 & 0 & 0 & 0 & 0 & 0 & 0 & F \\
\hline$D_{2} 0.125$ & F & 0 & F & 0 & 0 & 0 & 0 & 0 & 0 & 0 & 0 & 0 & 0 & 0 & 0 & F \\
\hline$D_{3} 0.125$ & F & o & 0 & F & 0 & 0 & 0 & 0 & 0 & 0 & 0 & 0 & 0 & 0 & 0 & F \\
\hline$D_{4} 0.125$ & F & o & 0 & 0 & F & 0 & 0 & 0 & o & 0 & o & o & 0 & 0 & 0 & $\mathbf{F}$ \\
\hline$D_{5} 0.125$ & F & o & 0 & 0 & 0 & $\mathbf{F}$ & 0 & 0 & 0 & 0 & o & $\begin{array}{ll}0 \\
\end{array}$ & 0 & 0 & 0 & F \\
\hline $\mathrm{D}_{6} \mathbf{0 . 1 2 5}$ & F & o & 0 & 0 & 0 & 0 & F & 0 & 0 & 0 & 0 & 0 & 0 & 0 & 0 & F \\
\hline $\mathrm{D}_{7} \mathbf{0 . 1 2 5}$ & F & 0 & 0 & 0 & 0 & 0 & 0 & F & 0 & 0 & 0 & 0 & 0 & 0 & 0 & F \\
\hline$D_{8} 0.125$ & F & 0 & 0 & 0 & 0 & 0 & 0 & 0 & F & 0 & 0 & 0 & 0 & 0 & 0 & F \\
\hline $\mathrm{D}, 0.125$ & F & 0 & 0 & 0 & 0 & 0 & 0 & 0 & 0 & $\mathbf{F}$ & 0 & $\begin{array}{ll}0 \\
\end{array}$ & 0 & 0 & 0 & F \\
\hline $\mathrm{D}_{10} 0.125$ & F & 0 & 0 & 0 & 0 & 0 & 0 & 0 & 0 & 0 & $\mathbf{F}$ & 0 & 0 & 0 & 0 & $\mathbf{F}$ \\
\hline $\mathrm{D}_{11} 0.125$ & F & 0 & 0 & 0 & 0 & 0 & 0 & 0 & 0 & 0 & 0 & F & 0 & 0 & 0 & $\mathbf{F}$ \\
\hline $\mathbf{D}_{12} 0.125$ & F & 0 & 0 & 0 & 0 & 0 & 0 & 0 & o & 0 & o & 0 & $\mathbf{F}$ & o & 0 & $\mathbf{F}$ \\
\hline $\mathrm{D}_{13} 0.125$ & F & o & 0 & 0 & 0 & 0 & 0 & 0 & 0 & 0 & 0 & 0 & 0 & $\mathbf{F}$ & 0 & $\mathbf{F}$ \\
\hline $\mathrm{D}_{14} 0.125$ & F & 0 & 0 & 0 & 0 & 0 & 0 & 0 & 0 & 0 & 0 & 0 & 0 & 0 & F & F \\
\hline
\end{tabular}

Generally, in order to find the stability of the structure after doping, the dopant formation energy $\left(E_{f}\right)$ simply refers to the energy needed to insert one or more fluorine atom into the supercell with a chemical potential $\mu_{F}$ after removing one or more Oxygen atom with a chemical potential $\mu_{O}$ from the same position, the formation energy was calculated using equation (1) [34].

$E_{f}=E_{\text {total }}\left(\mathrm{MoO}_{2}+F\right)-E_{\text {total }}\left(\mathrm{MoO}_{2}\right)+\mu_{O}-\mu_{F}$

Where $E_{f}=$ formation energy $(\mathrm{eV}), E_{\text {total }}\left(\mathrm{MoO}_{2}+\mathrm{F}\right)=$ total energy of Hexagonal Molybdenum dioxide doped fluorine $(\mathrm{eV}), E_{\text {total }}\left(\mathrm{MoO}_{2}\right)=$ total energy of pure molybdenum dioxide, $\mu_{0}=$ chemical potential per atom of oxygen, $\mu_{F}=$ chemical potential per atom of fluorine. The chemical potentials were numerically calculated as the total energy per atom in the bulk system. All the calculated formation energies were tabulated in the Table IV below.

TABLE IV FORMATION ENERGY OF PURE AND DOPED MOLYBDENUM DIOXIDE

\begin{tabular}{|l|l|l|l|l|}
\hline \multicolumn{1}{|c|}{ Compound } & Total Energy (eV) & Chemical potential & Free Atom & $\begin{array}{c}\text { Formation Energy } \\
(\mathrm{eV})\end{array}$ \\
\hline Pure $\mathrm{MoO}_{2}$ & -1631.024 & -67.959 & 3 & Nil \\
\hline $4.17 \%$ of Fluorine & -1647.430 & -68.643 & 3 & 232.5 \\
\hline $8.33 \%$ of Fluorine & -1663.530 & -69.326 & 4 & 463.0 \\
\hline $12.5 \%$ of Fluorine & -1680.306 & -70.013 & 5 & 698.5 \\
\hline
\end{tabular}

Table IV is the calculated formation energies of $4.17 \%$, $8.33 \%$, and $12.5 \%$ of fluorine on Hexagonal Molybdenum dioxide which are $232.5 \mathrm{eV}, 463.0 \mathrm{eV}$, and $698.5 \mathrm{eV}$ respectively. The table shows the variation of energy based on the increase in the doping concentration, the formation energy increases with the increase in the doping concentration which led to having the breakage of bond on structure. It was observed from Fig. 1( $a$ and $b$ ) that the structure of pure Hexagonal Molybdenum dioxide and $4.17 \%$ of fluorine on Hexagonal Molybdenum dioxide has three (3) free atoms, 
which maintain the stability of the structure, but when the doping concentration were increased, the number of free atoms increases from three (3) to four (4) in Fig. 1c for 8.33\% of fluorine and five (5) free atoms in Fig. 1d for $12.5 \%$ of fluorine. The formation energy of the doped compound of $4.17 \%$ of fluorine is $232.5 \mathrm{eV}$ which is more stable than $8.33 \%$, and $12.5 \%$ of fluorine with four (4), and five (5), free atoms respectively.
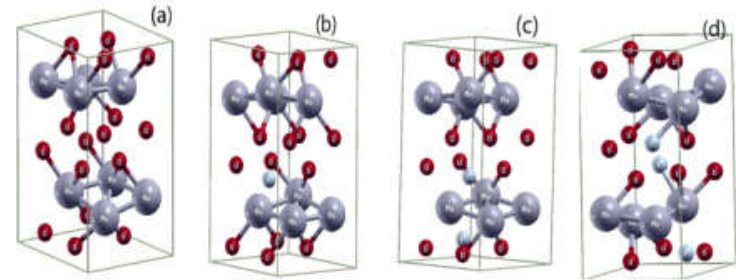

Fig. 1 (a) pure Molybdenum dioxide $\left(\mathrm{MoO}_{2}\right)$ (b) $4.17 \%$ of fluorine doped molybdenum dioxide $\left(\mathrm{F} . \mathrm{MoO}_{2}\right)$ (c) $8.33 \%$ of fluorine doped molybdenum dioxide $\left(\mathrm{F} . \mathrm{MoO}_{2}\right)$ (d) $12.5 \%$ of fluorine doped molybdenum dioxide $\left(\mathrm{F} . \mathrm{MoO}_{2}\right)$.

Table V shows the bond length of pure Hexagonal Molybdenum dioxide and Fluorine doped Hexagonal Molybdenum dioxide with different doping concentration. It was observed that $4.17 \%$ of fluorine increases in bond length when doped with pure Hexagonal molybdenum dioxide from $2.2505-2.3030 \mathrm{pm}$ which indicates a greater stability of the compound, because the bond length increases across a period and decreases down a group, similarly when doped with $8.33 \%$ and $12.5 \%$ of fluorine doped Hexagonal Molybdenum dioxide shows a decrease in bond length from 2.2698 $2.2099 \mathrm{pm}$ respectively, which shows the instability in the compound. The more atom holding together the greater the stability of the compounds and the bond length is inversely related to the bond energy. in this case, the four (4) and five (5) free atoms of $8.33 \%$, and $12.5 \%$ clearly shows the instability of the structures, and make $4.17 \%$ of fluorine on Hexagonal molybdenum dioxide be more stable.

TABLE V BOND LENGTH OF PURE AND DOPED MOLYBDENUM DIOXIDE

\begin{tabular}{|c|l|l|}
\hline Compound & \multicolumn{1}{|c|}{ \% of Dopant } & Bond Length $(\hat{A})$ \\
\hline $\mathrm{MoO}_{2}$ & Nil & 2.2505 \\
\hline $\mathrm{MoO}_{2}$ & $4.17 \%$ & 2.3030 \\
\hline $\mathrm{MoO}_{2}$ & $8.33 \%$ & 2.2698 \\
\hline $\mathrm{MoO}_{2}$ & $12.5 \%$ & 2.2099 \\
\hline
\end{tabular}

\section{CONCLUSION}

In conclusion, using the first-principles calculations, we have studied the effect of doping concentration on structural stability of the fluorine doped Hexagonal $\mathrm{MoO}_{2}$. The Doped structures with $\mathrm{F}$ replacing several distinct Oxygen at different doping concentrations $(4.17 \%, 8.33 \%$ and $12.5 \%)$ sites were looked into. From the calculated formation energies, the most stable structure among the $\mathrm{F}$ doped system is found to be at $4.17 \%$ doping concentration. The formations energies for doping $\mathrm{H}-\mathrm{MoO}_{2}$ with $\mathrm{F}$ are $17.09,34.173$ and $51.336 \mathrm{eV}$ at $4.17 \%, 8.33 \%$ and $12.5 \%$ doping concentration. respectively. Confirming for stability at $4.17 \%$ due to a less formation energies value.

\section{References}

[1] X. Li et al., "Large-area synthesis of high-quality and uniform graphene films on copper foils" Science, vol. 324, no. 5932, pp. 1312-1314, 2009.

[2] Q. Ji, Y. Zhang, Y. Zhang, and Z. Liu, "Chemical vapour deposition of group-VIB metal dichalcogenide monolayers: engineered substrates from amorphous to single crystalline" Chem. Soc. Rev., vol. 44, no. 9, pp. 2587-2602, 2015.

[3] J. Ji et al., "Two-Dimensional Monocrystalline Antimoene Layers Grown by Van der Waals Epitaxy", Nat. Comm., vol. 7, pp. 13352, 2016.

[4] L. K. Li et al., "Black Phosphorus Field-Effect Transistors", Nat. Nanotech., vol. 9, pp. 372-377, 2014.

[5] C. Xu et al., "Large-area high-quality 2D ultrathin Mo $2 \mathrm{C}$ superconducting crystals. Nat. mat., vol. 14, no. 11, pp. 1135-1141, 2015.

[6] C. M. Orofeo, S. Suzuki, and H. Hibino, "Ultrathin chemical vapor deposition (CVD)-grown hexagonal boron nitride as a high-quality dielectric for tunnelling devices on rigid and flexible substrates", The J. of Phy. Chem. C, vol. 118, no. 6, pp. 3340-3346, 2014.

[7] G. Fiori et al., "Electronics based on twodimensional materials", Nat. Nanotech., vol 9, 2014.

[8] W. Cheng, E. Baudrin, B. Dunn, and J. I. Zink, "Synthesis and electrochromic properties of mesoporous tungsten oxide Basis of a presentation given at Materials Discussion no. 3, 26-29 September, 2000, University of Cambridge, UK. J. of Mat. Chem., vo. 11, no.1, pp. 92-97, 2001.

[9] J. G., Lu, P., Chang, and Z. Fan, "Quasi-onedimensional metal oxide materials-Synthesis, properties and applications", Mat. Sci. and Eng. R: Rep., vol. 52, no. 1-3, pp. 49-91, 2006.

[10]D., Hirai, E. Climent-Pascual, and R. J. Cava, "Superconductivity in WO 2.6 F 0.4 synthesized by reaction of WO 3 with Teflon", Phy. Rev. B, vol. 84, no. 17, pp. 174519, 2011.

[11] L. C. Yang, Q. S. Gao, Y. H. Zhang, Y. Tang, and Y. P. Wu, "Tremella-like molybdenum dioxide consisting of nanosheets as an anode material for lithium ion battery", Electrochem. (b), 2008.

[12] Y. Shi et al., "Ordered mesoporous metallic $\mathrm{MoO}_{2}$ materials with highly reversible lithium storage capacity", Nano lett., vol. 9, no. 12, pp. 4215-4220, 2009. 
[13] L. C. Yang, Q. S. Gao, Y. H. Zhang, Y. Tang, and Y. P. Wu, "Tremella-like molybdenum dioxide consisting of nanosheets as an anode material for lithium ion battery", Electrochem. (b).

[14]P. Reinhardt, and B. A. Hess, "Electronic and geometrical structure of rutile surfaces", Phy. Rev. $B$, vol. 50, no. 16, pp. 12015, 1994.

[15]H. Wu et al., "Ultrathin Molybdenum Dioxide Nanosheets as Uniform and Reusable SurfaceEnhanced Raman Spectroscopy Substrates with High Sensitivity", Small, vol. 14, no. 37, pp. 1802276 , 2018.

[16]R. Tokarz-Sobieraj, R. Gryboś, and M. Witko, "Electronic structure of $\mathrm{MoO}_{2} /$. DFT periodic and cluster model studies", Appl. Cataly. A: General, vol. 391, no. 1-2, pp. 137-143, 2011.

[17] Y. Sun, X. Hu, W. Luo, and Y. Huang, "Selfassembled hierarchical $\mathrm{MoO}_{2}$ /graphene nanoarchitectures and their application as a highperformance anode material for lithium-ion batteries", ACS nano, vol. 5, no. 9, pp. 7100-7107, 2011.

[18]L. Yang et al., "Porous metallic $\mathrm{MoO}_{2}$-supported MoS 2 nanosheets for enhanced electrocatalytic activity in the hydrogen evolution reaction", Nanoscale, vol. 7, no. 12, pp. 5203-5208, 2015.

[19]M. Aryal, K. Trivedi, and W. Hu, "Nanoconfinement induced chain alignment in ordered P3HT nanostructures defined by nanoimprint lithography", ACS nano, vol. 3, no. 10, pp. 30853090, 2009.

[20]Q. Zhang et al., "A metallic molybdenum dioxide with high stability for surface enhanced Raman spectroscopy", Nat. comm., vol. 8, no. 1, pp. 1-9, 2017.

[21] A. Katrib, P. Leflaive, L. Hilaire, and G. Maire, "Molybdenum based catalysts. I. $\mathrm{MoO}_{2}$ as the active species in the reforming of hydrocarbons. Cataly. Lett., vol. 38, no. 1, pp. 95-99 (c), 1996.

[22] S. O. Akande, A. Chroneos, M. Vasilopoulou, S. Kennou, and U. Schwingenschlögl, "Vacancy formation in $\mathrm{MoO}_{3}$ : hybrid density functional theory and photoemission experiments. $J$. of Mat. Chem. $C$, vol. 4, no. 40, pp. 9526-9531, 2016.

[23]N. Wazir et al., "Comparative Studies on TwoDimensional (2D) Rectangular and Hexagonal Molybdenum Dioxide Nanosheets with Different Thickness", Nanoscale res. Lett., vol. 15, no. 1, pp. $1-9,2020$

[24]H. H. Kung, "Transition metal oxides: surface chemistry and catalysis", Elsev.,1989.

[25] D. B. Rogers, R. D. Shannon, A. W. Sleight, and J. L. Gillson, "Crystal chemistry of metal dioxides with rutile-related structures", Inorg. Chem., vol. 8, no. 4, pp. 841-849, 1969.

[26] V. Eyert, R. Horny, K. H. Höck, and S. Horn, "Embedded Peierls instability and the electronic structure of MoO2", J. of Phy.: Cond. Matt., vol. 12, no. 23, pp. 4923, 2000.

[27]D. O. Scanlon, G. W. Watson, D. J. Payne, G. R., Atkinson, R. G. Egdell, and D. S. L. Law, "Theoretical and experimental study of the electronic structures of $\mathrm{MoO}_{3}$ and $\mathrm{MoO}_{2}$. The Journal of Physical Chemistry C, vol. 114, no. 10, pp. 46364645,2010

[28] M. Ghedira, C. Do-Dinh, M. Marezio, and J. Mercier, "The crystal structure of Mo0. 975Ti0. $025 \mathrm{O} 2$ between 24 and $900^{\circ}$ C", J. of Sol. St. Chem., vol. 59, no. 2 , pp. $159-167$.

[29]P. Giannozzi et al., "QUANTUM ESPRESSO: a modular and open-source software project for quantum simulations of materials", J. of Phy.: Cond. Matt., vol. 21, no. 39, pp. 395502.

[30] J. P. Pardew, K. Burke, and M. Emzerhof, "Generalized gradient approximation made simple", Phy. Rev. Lett., vol. 77, no. 18, pp. 3865, 1996.

[31] W. J. Wang et al., "Metal-insulator-semiconductorinsulator-metal structured titanium dioxide ultraviolet photodetector", J. of Phy. D: Appl. Phy., vol. 43, no.4, pp. 045102 (a), 2010.

[32] X. Fan, F. Wang, Z. Chu, L. Chen, C. Zhang, and D. Zou, "Conductive mesh based flexible dye-sensitized solar cells", Appl. Phy. Lett., vol. 90, no. 7, pp. $073501,2007$.

[33]D. S. Parker et al., "Low temperature formation of naphthalene and its role in the synthesis of PAHs (polycyclic aromatic hydrocarbons) in the interstellar medium", Proc. of the Nat. Acad. of Sci., vol. 109, no. 1, pp. $53-58,2012$.

[34] C. Zhang, C. L. Wang, J. C. Li, K. Yang, Y. F., Zhang, and Q. Z. Wu, "Substitutional position and insulator-to-metal transition in Nb-doped SrTiO3. Mat. Chem. and Phy., vol. 107, no. 2-3, pp. 215-219, 2008. 